\title{
Transcriptome analysis of callus from Picea balfouriana
}

\author{
Qingfen Li, Shougong Zhang and Junhui Wang*
}

\begin{abstract}
Background: Picea likiangensis var. balfouriana (Rehd. et Wils.) Hillier ex Slavin (also known as Picea balfouriana) is an ecologically and economically important conifer that grows rapidly under optimum conditions and produces high-quality wood. It has a wide geographic distribution and is prevalent in southwest and eastern regions of China. Under suboptimal conditions, P. balfouriana grows slowly, which restricts its cultivation. Somatic embryogenesis has been used in the mass propagation of commercial species. However, low initiation rates are a common problem and the mechanisms involved in the induction of somatic embryogenesis are not fully understood. To understand the molecular mechanisms regulating somatic embryogenesis in P. balfouriana, high-throughput RNA-seq technology was used to investigate the transcriptomes of embryogenic and non-embryogenic tissues from three $P$. balfouriana genotypes. We compared the genes expressed in these tissues to identify molecular markers with embryogenic potential.

Results: A total of 55,078,846 nucleotide sequence reads were obtained for the embryogenic and non-embryogenic tissues of $P$. balfouriana, and $49.56 \%$ of them uniquely matched 22,295 (84.3\%) of the 26,437 genes in the Picea abies genome database (Nature 497: 579-584, 2013). Differential gene expression analysis identified 1,418 differentially expressed genes (false discovery rate $<0.0001$; fold change $\geq 2$ ) in the embryogenic tissues relative to the non-embryogenic tissues, including 431 significantly upregulated and 987 significantly downregulated genes. KEGG (Kyoto Encyclopedia of Genes and Genomes) pathway analysis revealed that the most significantly altered genes were involved in plant hormone signal transduction, metabolic pathways (starch and sucrose metabolism), and phenylalanine metabolism.

Conclusions: We found that the initiation of embryogenic tissues affected gene expression in many KEGG pathways, but predominantly in plant hormone signal transduction, plant-pathogen interaction, and starch and sucrose metabolism. The changes in multiple pathways related to induction in the $P$. balfouriana embryogenic tissues described here, will contribute to a more comprehensive understanding of the mechanisms involved in the initiation of somatic embryogenesis. Additionally, we found that somatic embryogenesis receptor kinase (SERK), arabinogalactan proteins, and members of the WUS-related homeobox protein family may play important roles and could act as molecular markers in the early stage of somatic embryogenesis, as reported previously.
\end{abstract}

Keywords: Picea balfouriana, Somatic embryogenesis, Embryogenic tissue, Non-embryogenic tissue

\section{Background}

Picea balfouriana grows in a specific type of forest ecosystem in southwest China and is an eastern species on the Tibetan plateau. For rapid growth and production of high-quality wood, many plantations selected $P$. balfouriana as one of the main species for afforestation. Seedlings of $P$. balfouriana are produced primarily by sexual propagation;

\footnotetext{
*Correspondence: wangjh@caf.ac.cn

State Key Laboratory of Forest Genetics and Tree Breeding, Research Institute of Forestry, Chinese Academy of Forestry, Number 1 of Dongxiaofu in Haidian District, Beijing, China
}

however, the seeds set late in the season, and the seedlings grow very slowly during the early phases.

Propagation by somatic embryogenesis (SE) is the primary technology used for many conifer biotechnology products, including the development of transgenic trees. The SE system for most spruce (Picea spp.) and some pine (Pinus spp.) species is sufficiently refined for commercial use; however, for many economically important conifer species, the initiation frequency of SE is insufficient for commercial applications. In recent years, much research has been focused on the development and optimization of protocols for the induction and maturation of somatic

\section{Biomed Central}

(c) 2014 Li et al.; licensee BioMed Central Ltd. This is an Open Access article distributed under the terms of the Creative Commons Attribution License (http://creativecommons.org/licenses/by/2.0), which permits unrestricted use, distribution, and reproduction in any medium, provided the original work is properly credited. 
embryos of coniferous species [1]. The developmental stage of zygotic embryos, the contents of the growth or culture medium, the type and concentration of plant growth regulators, and culture condition factors such as $\mathrm{pH}$, agar type, and nitrogen level can affect the generation of embryogenic tissue (ET). Therefore, understanding and balancing these factors is crucial for increasing the initiation rate of SE. A complete understanding of the genes involved in the development of ET and non-embryogenic tissue (NET) will improve the understanding of the initiation process in embryogenic tissue.

Cellular identity and function are determined by the transcriptome, i.e. the complete repertoire of expressed RNA transcripts. Transcriptome profiling is a powerful method that has been used widely to assess the relative importance of gene products in cells, tissues, organisms, or under different conditions. Next-generation sequencing (NGS) technology is a cutting-edge approach for highthroughput sequence determination, which has dramatically improved the efficiency and speed of gene discovery $[2,3]$. For example, Illumina sequencing technology generates more than one billion bases of high-quality DNA sequence per run at less than $1 \%$ of the cost of capillary-based methods [4]. Thus, NGS has significantly accelerated and improved the sensitivity of gene-expression profiling, and is expected to boost collaborative and comparative genomics studies [5,6]. Illumina sequencing of transcriptomes for organisms with completed genomes confirmed that the relatively short reads produced by this methodology can be assembled effectively and used for gene discovery and comparisons of gene expression profiles $[7,8]$. Despite the obvious potential, NGS methods have not yet been used in P. balfouriana.

The high-throughput RNA-seq methodology involves whole-transcriptome shotgun sequencing, in which mRNA or cDNA is mechanically fragmented to produce overlapping short fragments that cover the entire transcriptome [9-11]. By mapping the RNA-seq reads to a reference genome, gene assignments and large-scale functional annotation of genes can be carried out. Further, compared with DNA microarray data, RNA-seq data can be used to detect and quantify RNAs that are expressed at very low levels [12]. Additionally, Gene Ontology (GO) analysis of the annotated genes can lead to a deeper understanding of the functions of the genes in cells, thereby providing sensitive and accurate profiling of the transcriptome and a description of gene function that resembles the biology of the cell [13].

Very few studies reporting the mechanism of initiation during SE have been published so far. The main objective of the present study was to identify differentially expressed genes in ETs and NETs, independent of genotype, through RNA-seq analysis and mapping to the reference Picea abies genome [14]. The results of this work may provide fundamental insights into the early SE in P. balfouriana.

\section{Results}

RNA-seq library sequencing

Six P. balfouriana RNA-seq libraries were produced and sequenced; namely, the ETs and NETs from three genotypes. In each library, 11.7-12.5 million clean reads were obtained after the low-quality reads were filtered out (Table 1). Among the clean reads, 8.4-10.9 million (71.24-87.31\%) were mapped to known genes (Table 1).

Approximately 19,000 genes (short read mapped to reference genome) were detected in each library, accounting for more than $70 \%$ of the 26,437 genes in the P. abies reference genome database [14] (Table 2). Overall, 22,295 unique genes were expressed among the six libraries (Additional file 1), which gives a mapping coverage of $84.3 \%$, conferring high confidence in the RNA-seq results. The mean coverage of all genes was above $60 \%$, with the highest coverage at $99.96 \%$. GO terms were assigned to the mapped genes and an enrichment analysis of the GO terms showed that intracellular organelle part, anion binding, and primary metabolic process were dominant in the cellular component, molecular function, and biological process categories, respectively. Some of the genes assigned primary metabolic process terms were associated with KEGG pathways involved in purine metabolism, RNA polymerase, pyrimidine metabolism, inositol phosphate metabolism and phenylalanine, and tyrosine and tryptophan biosynthesis (Additional file 2).

\section{Uniform genes with consistent fold-changes in ETs and NETs from the three genotypes}

After two-factor analysis of variance, 4,734 genes with significantly different effects for "embryonic vs. nonembryonic" $(\mathrm{P}<0.05)$ and insignificant effects for "genotype" ( $P>0.05)$ were selected (Additional file 3$)$. Then, the selected genes were analyzed using DESeq(version 2.14) to test for differentially expressed genes (DEGs) in ETs and NETs, independent of genotype (false discovery rate $($ FDR $<0.001$, fold change $\geq 2$ ). Ultimately, 1,418 DEGs between ET and NET were identified, among which 431 were upregulated and 987 were downregulated (Additional file 4). Some of the DEGs were associated with SE (germin-like proteins, abscisic acid receptor, cytochrome P450, and chitinases). About one-quarter of the genes were upregulated by $\geq 2$-fold, and about 1,000 genes were downregulated by $\geq 2$-fold.

Oxidoreductase activity, cell wall, and apoplast were the most enriched GO terms, while biosynthesis of secondary metabolites, metabolic pathways, pentose and glucuronate interconversion, starch and sucrose metabolism, phenylpropanoid biosynthesis, plant hormone signal transduction, flavonoid biosynthesis, phenylalanine metabolism, ether lipid 
Table 1 Mapping of Picea balfouriana RNA-seq library reads to the Picea abies reference genome database

\begin{tabular}{lcccccc}
\hline Classification & ET Genotype1 & NET Genotype 1 & ET Genotype 2 & NET Genotype 2 & ET Genotype 3 & NET Genotype 3 \\
\hline Raw reads & $12,519,224$ & $11,703,983$ & $12,150,702$ & $11,734,531$ & $12,150,562$ & $12,280,320$ \\
Base pairs & $613,419,76$ & $573,495,167$ & $595,384,398$ & $574,992,019$ & $595,377,538$ & $601,735,680$ \\
$\%$ & 100.00 & 100.00 & 100.00 & 100.00 & 100.00 & 100.00 \\
Mapped reads & $5,713,055$ & $5,044,017$ & $5,590,502$ & $4,359,900$ & $5,519,889$ & $5,456,021$ \\
\% & 45.63 & 43.10 & 46.01 & 37.15 & 45.43 & 44.43 \\
Perfect match & $3,923,702$ & $3,401,759$ & $3,865,351$ & $2,947,585$ & $3,870,249$ & $3,674,461$ \\
$\%$ & 31.34 & 29.06 & 31.81 & 25.12 & 31.85 & 29.92 \\
$<=2$ bp mismatch & $1,789,353$ & $1,642,258$ & $1,725,151$ & $1,412,315$ & $1,649,640$ & $1,781,560$ \\
\% & 14.29 & 14.03 & 14.20 & 12.04 & 13.58 & 14.51 \\
Unique match & $5,374,869$ & $47,721,812$ & $5,262,180$ & $4,081,332$ & $5,214,938$ & $5,117,424$ \\
\% & 42.93 & 40.34 & 43.31 & 34.78 & 42.92 & 41.67 \\
Multi-position match & 338,186 & 322,205 & 328,322 & 278,568 & 304,951 & 338,597 \\
\% & 2.70 & 2.75 & 2.70 & 12.42 & 2.51 & 6.76 \\
Unmapped reads & $6,806,169$ & $6,659,966$ & $6,560,200$ & $7,374,631$ & $6,630,673$ & 54.824 \\
\% & 54.37 & 56.90 & 53.99 & 62.85 & 55.57 \\
\hline
\end{tabular}

ET, embryogenic tissue; NET, non-embryogenic tissue.

metabolism, and propanoate metabolism were the most overrepresented KEGG pathways among genes related to embryogenic competence (Additional file 5). These findings will be very important for further understanding the intracellular signaling mechanisms of early SE in conifers.

\section{Expression of housekeeping genes in the different tissue samples of $P$. balfouriana}

The NormFinder algorithm uses a model-based approach to estimate alterations in the expression of housekeeping genes [15]. The algorithm also estimates variations across subgroups and avoids the artificial selection of co-regulated genes. The results of the NormFinder analysis of the genes detected in the six libraries show that WS0109_C05 [TAIR: At1g29260] and 18S rRNA were predicted to be the most stable housekeeping genes (Table 3).

BestKeeper measures stability using a pair-wise correlation analysis of all pairs of candidate genes and calculates the geometric mean of the best candidates [16,17]. A preliminary analysis based on the inspection of raw $\mathrm{Ct}$ values estimated the variation of four housekeeping genes (WS00912.B21_N13, WS0109_C05, 18S rRNA, and tubulin) to be compatible with an overall stability in gene expression (Table 4). The standard deviation values were $<1$. The four housekeeping genes were used to calculate the BestKeeper index. BestKeeper allows a comparative analysis across internal reference genes by estimating correlations in the expression levels between all the possible candidates. Highly correlated control genes are combined into an index, and the pair-wise correlation between genes and the correlation between each gene and the index are also calculated. The results describe the consistency between the index and each internal reference gene. The four control genes tested in our analysis correlated well one with one another and with the NormFinder index (Table 3). The best correlation between one internal reference gene and the BestKeeper index was for WS0109_C05 $(r=0.947)$. The statistically significant correlation for WS0109_C05 with the BestKeeper index appeared to be consistent with the good performance of this housekeeping gene as assessed by NormFinder.

\section{Quantitative real-time reverse transcription-polymerase chain reaction (qRT- PCR) analysis}

The peroxisomal targeting signal receptor WS0109_C05 displayed constitutive expression in all the analyses performed using the P. balfouriana RNA-seq data; therefore, it was used as an internal control for the normalization of gene expression levels $(\Delta \mathrm{CT})$. Total RNA from the same six tissue samples that were used for the RNA-seq analysis

Table 2 Genes found in Picea balfouriana that uniquely match genes in the Picea abies reference genome

\begin{tabular}{lccccccc}
\hline & ET Genotype 1 & NET Genotype 1 & ET Genotype 2 & NET Genotype 2 & ET Genotype 3 & NET Genotype 3 \\
\hline Uniquely matched genes & 18865 & 19256 & 19432 & 19143 & 19116 & 19287 \\
Percentage & $71.36 \%$ & $72.84 \%$ & $73.50 \%$ & $72.41 \%$ & $72.31 \%$ & $72.95 \%$ \\
\hline
\end{tabular}

ET, embryogenic tissue; NET, non-embrygeonic tissue. 
Table 3 Ranking of Picea balfouriana candidate reference genes generated by NormFinder

\begin{tabular}{lcc}
\hline Gene name & Stability value & Standard error \\
\hline WS00912.B21_N13 & 0.403 & 0.268 \\
WS0109_C05 & 0.282 & 0.311 \\
18S rRNA & 0.923 & 0.324 \\
Tubulin & 0.779 & 0.295 \\
\hline
\end{tabular}

were used as templates for the qRT-PCR analysis (Table 5). For most of the genes, the transcript fold-ratios determined by qRT-PCR were approximately the same as those estimated from the RNA-seq data, indicating the reliability of the RNA-seq in P. balfouriana.

\section{Discussion}

Most early studies on the initiation mechanism of SE in $P$. balfouriana focused mainly on different genes between ET and NET from just one cell line. It has been suggested that genotype may have an important effect on initiation of ET. In this study, we demonstrated that many genes were influenced by genotype, so we filtered them out and selected the genes that were related only to embryogenic ability. Six RNA-seq libraries were created to analyze the DEGs in ET and NET, yielding a total of 22,295 genes. Among them, 9,988 (44.8\%) and 13,135 (58.9\%) were enriched in GO terms and KEGG pathways, respectively. Our transcriptome and gene expression profiling data will greatly enrich the current knowledge for the genus Picea, and will contribute to the database for conifer species. These data will also help promote research on the identification of novel genes related to embryogenic competence.

Several genes involved in the response to phytohormone and hormone-mediated signaling pathways in ETs were strongly upregulated (Additional file 4); for example, the genes that encode auxin-induced proteins. The initiation of embryogenesis in somatic tissues requires auxins, especially in conifer species. Exogenous hormones and culture-related stress has been reported to play important roles in triggering SE, which presumably results from the expression of genes involved in cell division, cell polarization, and the regulation of signal transduction pathways. Several auxin-inducible genes that are involved in cellular changes, such as activation of cell division, have been cloned [18,19]. Bögre et al. [20] used auxin-responsive genes as molecular markers to distinguish embryogenic from non-embryogenic genotypes of Medicago sativa. Our results indicated that most of the DEGs were involved in plant-pathogen interaction, metabolic pathways (starch and sucrose metabolism), and plant hormone signal transduction (Additional file 5) and, therefore, they may play essential roles in the initiation of SE.

A number of genes encoding membrane-localized, leucine-rich, repeat receptor-like kinases, such as IMK2 [Swiss-Prot:Q9SCT4], were among the DEGs. The somatic embryogenesis receptor kinase (SERK) gene encodes a leucine-rich repeat receptor-like kinase that plays an important role in plant signaling pathways. The SERK gene was first identified in carrot (Daucus carota) suspension cultures where it was specifically expressed in cells that developed into somatic embryos [21]. SERK has been linked to SE in a number of species, including Dactylis glomerata [22], Arabidopsis thaliana [23], Medicago truncatula [24], sunflower (Helianthus annuus) [25], Ocotea catharinensis [26], Citrus unshiu [27], and Theobroma cacao [28]. There is evidence that SERK genes are required for embryo initiation in embryogenic cells $[21,23,29]$. In D. glomerata, and A. thaliana, SERKs are characteristic markers of embryogenic cell cultures and somatic embryos [21-23].

Several arabinogalactan proteins (AGPs) were upregulated in ETs compared with NETs, such as FLA7 [Swiss-Prot:Q9SJ81]. AGPs have been implicated in five fundamental cellular processes: cell proliferation, cell

Table 4 Statistical output from the BestKeeper analysis for the Picea balfouriana candidate reference genes

\begin{tabular}{lcccc}
\hline & WS00912.B21_N13 & WS0109_C05 & 18SrRNA & Tubulin \\
\hline$N$ & 6 & 6 & 6 & 13.12 \\
G Mean [Ct] & 28.03 & 27.57 & 13.13 & 24.37 \\
A Mean [Ct] & 28.06 & 27.58 & 12.33 & 24.4 \\
Min [Ct] & 26.21 & 26.38 & 13.81 & 22.62 \\
Max [Ct] & 29.78 & 28.79 & 0.56 & 25.68 \\
SD $[ \pm C t]$ & 0.85 & 0.58 & 4.26 & 1.06 \\
CV [\% Ct] & 3.02 & 2.09 & -0.014 & 0.35 \\
Coeff. of corr. [r] & 0.85 & 0.947 & 0.978 & 0.203 \\
$P$-value & 0.032 & $0.004^{*}$ &
\end{tabular}

$\mathrm{N}$, number of samples; G Mean [Ct], geometric mean of the $\mathrm{Ct}$; A Mean [Ct], arithmetic mean of the $\mathrm{Ct}$; Min [Ct] and Max [Ct], extreme values of the Ct; SD [ $\pm \mathrm{Ct}$, standard deviation of the $\mathrm{Ct} ; \mathrm{CV}[\% \mathrm{Ct}$ ], coefficient of variance expressed as percentage on the Ct level. Coeff. of corr. [r], coefficient of correlation. *Indicates the best correlation between the control genes and the BestKeeper index. 
Table 5 Comparison of RNA-seq data with expression data from qRT-PCR

\begin{tabular}{|c|c|c|c|c|c|c|}
\hline NCBI gene ID & UniProt_ID & Description & Method & $\begin{array}{l}\text { Expression } \\
\text { level in ET }\end{array}$ & $\begin{array}{c}\text { Expression } \\
\text { level in NET }\end{array}$ & Fold change \\
\hline \multirow[t]{4}{*}{840475} & \multirow[t]{4}{*}{ Swiss-Prot:Q9LP24 } & \multirow{4}{*}{$\begin{array}{l}\text { Leucine-rich repeat } \\
\text { receptor-like protein kinase }\end{array}$} & \multirow[t]{2}{*}{ RNA-seq } & Log-cpm in ET & Log-cpm in NET & log2 ratio (ET/NET) \\
\hline & & & & 605.5 & 3640.6 & 2.6 \\
\hline & & & \multirow[t]{2}{*}{ qRT-PCR } & $\mathrm{ET}(\Delta \mathrm{CT})$ & $\operatorname{NET}(\Delta C T)$ & $2^{-\Delta \Delta C T}$ \\
\hline & & & & 9.6 & 4.6 & 2.1 \\
\hline \multirow[t]{4}{*}{817958} & \multirow[t]{4}{*}{ Swiss-Prot:Q6X7J4 } & \multirow[t]{4}{*}{ WUSCHEL-related homeobox 9} & \multirow[t]{2}{*}{ RNA-seq } & Log-cpm in ET & Log-cpm in NET & $\log 2$ ratio (ET/NET) \\
\hline & & & & 155.7412756 & 0.353625 & 8.8 \\
\hline & & & \multirow[t]{2}{*}{ qRT-PCR } & $\mathrm{ET}(\Delta \mathrm{CT})$ & $\operatorname{NET}(\Delta C T)$ & $2^{-\Delta \Delta C T}$ \\
\hline & & & & 3.0 & 1.3 & 3.4 \\
\hline \multirow[t]{4}{*}{831649} & \multirow[t]{4}{*}{ Swiss-Prot:Q8GY25 } & \multirow[t]{4}{*}{ WUSCHEL-related homeobox 12} & \multirow[t]{2}{*}{ RNA-seq } & Log-cpm in ET & Log-cpm in NET & $\log 2$ ratio (ET/NET) \\
\hline & & & & 136.2 & 1.8 & 6.3 \\
\hline & & & \multirow[t]{2}{*}{ qRT-PCR } & $\mathrm{ET}(\Delta \mathrm{CT})$ & $\operatorname{NET}(\Delta C T)$ & $2^{-\Delta \Delta C T}$ \\
\hline & & & & 0.7 & 0.1 & 1.5 \\
\hline
\end{tabular}

Three biological repeats were reverse transcribed and amplified independently. The raw threshold cycle (Ct) values were normalized against the WS0109_C05 standard to generate the normalized $\Delta \mathrm{Ct}$ values that were used to calculate the fold-change in expression in embryonic tissue (ET) and non-embryonic tissue (NET) of the three genotypes. Log-cpm, log-counts per million.

expansion, cell differentiation, programmed cell death, and cell-cell communication [30]. The involvement of AGPs in SE has been studied previously [31-37], and the role of AGPs in the establishment of embryogenic cell cultures and the influence of AGPs on cell development have also been examined. The presence of AGPs in seeds was found to increase the number of pro-embryogenic masses and the embryogenic potential [38-41]. Filonova et al. [42] reported the importance of intercellular communication for the acquisition of embryogenic competence, and observed that separation of Norway spruce cells by fractionation of suspension cultures inhibited the embryogenic process. AGPs restore differentiation potential after cell wall removal, and this restoration was reported to be more efficient with chitinase-cleaved forms of AGPs [36]. Based on these observations, we propose that complex interactions between cells and substances secreted in the medium of embryogenic cultures are essential to establish and maintain embryogenic competence in culture.

The qRT-PCR results confirmed the observed upregulation in ETs of two genes belonging to the WUS-related homeobox (WOX) family. Previous studies have reported the expression dynamics of $W O X$ and shown that it is highly expressed during early somatic embryo development, but declines as the embryo matures [43-45]. Haecker et al. [43] found that WOX8 and WOX9 were expressed in A. thaliana during embryo development from the very early stages and throughout development. Several WOX genes, including $W O X 2$, were reported to constitute potential markers of cell fate during early embryogenesis, suggesting that they might have important functions in early embryonic patterning [44]. Palovaara et al. [45] found that WOX2 played a fundamental role in early somatic embryo development in P. abies, possibly related to the regulation of cell division and/or differentiation in the embryos. It was also proposed that WOX 2 could be used as a molecular marker of embryogenic potential, but was not necessary for the regenerative capacity of cell lines [45]. Klimaszewska et al. [46] reported that WOX2 was expressed exclusively in the early stages of SE, and might be useful as a marker of embryogenic potential. WOX3, WOX8A, and WOX8B were all significantly expressed in proembryogenic masses in $P$. abies rather than in somatic embryos [47].

\section{Conclusions}

Although ET and NET can be discriminated by visual inspection, and molecular markers of embryogenic potential are useful, bioinformatics tools provide a powerful approach to identify DEGs in ETs and NETs. Here, we found gene expression patterns in ETs from $P$. balfouriana that identified changes in multiple pathways related to early $\mathrm{SE}$, such as plant hormone signal transduction, metabolic pathways (starch and sucrose metabolism), and phenylpropanoid biosynthesis. If these pathways could be modified to induce ETs with higher initiation rates that have competence to form normal somatic embryos they could be used to increase the initiation frequency of SE for commercial applications.

A large number of candidate genes, such as those that encode heat shock proteins and glutathione S-transferases, showed significant different expression in the ET libraries compared with their expression in the NET libraries; however, these results require further verification and characterization. Although the molecular functions of individual $P$. balfouriana genes and the associated signal 
transduction pathways often remained largely unknown, the RNA-seq analysis has provided valuable information about the induction of SE in $P$. balfouriana, which could promote further investigations into the detailed physiological initiation mechanisms of SE. This analysis represents a starting point for detailed functional studies; however, further experiments are required to expand on these results and to define the complex interaction networks and molecular mechanisms responsible for the induction of SE in P. balfouriana.

\section{Methods}

\section{Plant material}

ETs and NETs were collected from three genotypes of $P$. balfouriana (Figure 1), and initiated and maintained on $1 / 2 \mathrm{LM}$ basal medium [48], supplemented with $2.2 \mathrm{mg} \cdot \mathrm{L}^{-1}$ 2,4-dichlorophenoxyacetic acid (2,4-D), $1.1 \mathrm{mg} \cdot \mathrm{L}^{-1} \mathrm{BAP}$, $0.2 \mathrm{mg} \cdot \mathrm{L}^{-1} \mathrm{KT}, 1 \%$ sucrose, $1000 \mathrm{mg} \cdot \mathrm{L}^{-1}$ casein hydrolysate, $1 \%$ sucrose, $500 \mathrm{mg} \cdot \mathrm{L}^{-1}$ glutamine, and $0.2 \%$ gellan gum (Sigma, St. Louis, MO, USA).

\section{RNA isolation}

Total RNA was extracted immediately from fresh ET and NET samples, TRIZOL ${ }^{\oplus}$ reagent (Invitrogen, Carlsbad, CA, USA) was used to extract total RNA from 50 to $100 \mathrm{mg}$ of tissue, according to the manufacturer's instructions. The RNA samples were incubated for $30 \mathrm{~min}$ at $37^{\circ} \mathrm{C}$ with $10 \mathrm{U}$ of DNaseI (Takara, Dalian, China) to remove residual genomic DNA. The quality and quantity of the purified RNA were determined by measuring the absorbance at $260 \mathrm{~nm}$ and $280 \mathrm{~nm}$ (A260/A280) using a Nano-drop ${ }^{\oplus}$ ND-1000 spectrophotometer (LabTech, Holliston, MA, USA). The samples had an average RNA integrity number (RIN) of 8.9 based on a lab-on-chip analysis using a 2100 Bioanalyzer (Agilent Technologies, Santa Clara, CA, USA).

\section{RNA-seq library preparation and sequencing}

After extracting total RNA from the six samples, oligo (dT) magnetic beads were used to enrich the mRNA by removing rRNA. Fragmentation buffer was used to break
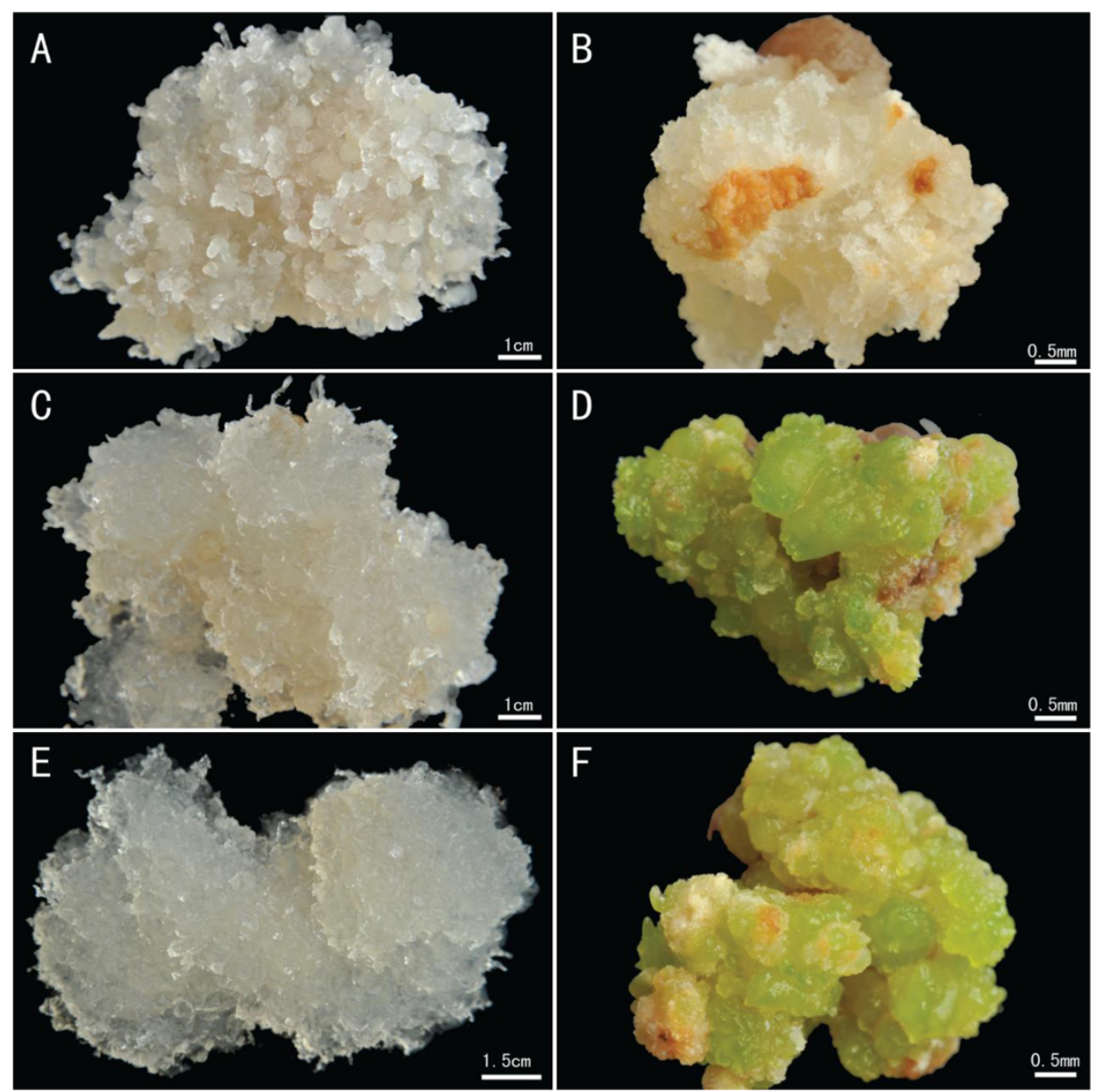

Figure 1 Embryonic tissue (ET) and non-embryonic tissue (NET) of three Picea balfouriana genotypes. (A) and (D) ET (bar $=1.0 \mathrm{~cm})$ and NET $($ bar $=1.5 \mathrm{~cm})$ of genotype 1, respectively; $(\mathbf{B})$ and $(\mathbf{E}) \mathrm{ET}(\mathrm{bar}=1.0 \mathrm{~cm})$ and NET $($ bar $=1.5 \mathrm{~cm})$ of genotype 2, respectively; (C) and $(\mathbf{F})$ ET $(\mathrm{bar}=1.0 \mathrm{~cm})$ and NET $(\mathrm{bar}=2.0 \mathrm{~cm})$ of genotype 3, respectively. 
the mRNA into short fragments (about 200-bp long), Then a random hexamer-primer was used to generate first-strand cDNA using the mRNA fragments as templates. Buffer, dNTPs, RNase $\mathrm{H}$, and DNA polymerase I were added to synthesize the second-strand cDNA. A QiaQuick PCR extraction kit (Qiagen, Hilden, Germany) was used to purify the double-strand cDNA, which was then washed with EB buffer for end repair and poly(A) addition. Finally, sequencing adaptors were ligated onto the fragments. Agarose gel electrophoresis was used to purify the fragments, which were then enriched by PCR amplification. The library products were sequenced using a Illumina HiSeqTM 2000 system (Illumina, San Diego, USA).

\section{Analysis and mapping of RNA-seq reads}

The sequenced raw data were filtered to remove lowquality tags (reads with unknown nucleotides "N"), empty reads (no read sequence between the adaptors), and reads with only one copy number (reads that might have resulted from sequencing errors). SOAPaligner/soap2 (version2.21) [49] was used to align the remaining clean reads to the sequences in the $P$. abies reference genome database, allowing up to two base mismatches. At most, one continuous gap or two mismatches were allowed in the high-quality part of a read. The mapped clean reads were designated as unambiguous clean reads. For twofactor analysis of variance, the number of unambiguous clean reads for each gene was calculated and normalized to log-counts per million using the latest version of the limma package in R [50]. The RNA-seq data consisted of a matrix of read counts $r_{g i}$, for RNA samples $i=1$ to $\mathrm{n}$, and genes $g=1$ to $\mathrm{G}$. If $R_{i}$ for the total number of mapped reads for sample $\mathrm{i}$ is defined as $R i=\sum_{g=1}^{G}{ }_{g i}$, then the logcounts per million (log-cpm) value for each count can be calculated as $y_{g a}=\log _{2}\left(\frac{r_{g i}+0.5}{R i+1.0} \times 10^{6}\right)$.

The counts were offset away from zero by adding 0.5 to avoid taking the log of zero, and to reduce the variability of log-cpm for low expression genes. The library size was offset by 1 to ensure that $\left(r_{g i}+0.5\right) /\left(R_{i}+1\right)$ was always less than 1 and greater than zero.

\section{Differentially expressed genes between the ET and NET libraries}

Two-factor analysis of variance for each gene, using two factors, "embryonic vs. non-embryonic" and "genotype", was performed in $\mathrm{R}$ (version 2.15.) to detect genes that were differentially expressed between the ET and NET libraries, and not affected by genotype. DESeq was also used to detect the DEGs between the ETs and NETs [51]. Finally, genes with significant effects from "embryonic vs. non-embryonic" $(\mathrm{P}<0.05)$ and insignificant effects from "genotype" $(\mathrm{P}>0.05)$ in two-factor analysis of variance were selected from the significantly changed genes detected by DESeq and considered as the DEGs between the ET and NET libraries. The FDRs of these DEGs were less than 0.001 and the absolute values of the $\log _{2}$ ratio were greater than 1 [52]. Briefly, if $R$ DEGs are selected and $S$ genes are genuinely differentially expressed while $V$ genes are false positives, and if the error ratio $(\mathrm{Q}=V / R)$ is required to remain below a cutoff (e.g. 0.001), then the FDR can be calculated according to the Benjamini and Hochberg algorithm [52] as: $F D R=E(Q)=E\{V /(V+S)\}=E(V / R)$.

In this study, a FDR of $\leq 0.001$ was used.

All the genes, including the DEGs, were used for the GO and KEGG ontology (KO) enrichment analyses. For the GO analysis, a corrected P-value of $<0.05$ was used as the threshold to determine significant enrichment of the gene sets. For $\mathrm{KO}$ enrichment analysis, a Q-value $\leq 0.05$ was used as the threshold to determine significant enrichment of the gene sets.

\section{Validation of DEGs by qRT-PCR}

Four reference housekeeping genes were selected for $P$. balfouriana and forward and reverse primers were designed as follows: WS00912.B21_N13 (5'-GTCGTG TGGATTGTCTCTGC-3'; 5' -ATGTATTCGAAGAGGA GGAATG-3'), WS0109_C05 (5'-AACTGTCATTGGA GTCCTGTAGG-3'; 5' -TGAGGGTTTGGGT AGTGA GA-3'), tubulin (PaTubF: 5'-CGTTACCTGCTGCCT GAG-3', PaTubR: 5'-G CTCTGTATTGCTGTGAAC C-3'), and 18S rRNA (Pa18SF:5'-CGGCGGATG TT GCTCTAAG-3'; Pa18SR:5'-TCTGTCAATCCTTACTA TGTCTGG-3'). Those gene has been used as a reference in other studies $[53,54]$. The most stable gene (WS0109_C05) was selected as the reference gene. Details of the qRT-PCR analysis of spruce transcripts has been described previously [55]. In brief, total RNA $(18 \mu \mathrm{g})$ from each sample was treated with DNase I (Invitrogen). The absence of DNA in the treated RNA samples (10 ng) was confirmed by PCR using the WS0109_C05 primers. Next, RNA (three reactions of $4 \mu \mathrm{g}$ each per sample) was reverse transcribed, and cDNA (10 ng) was analyzed by PCR in a total volume of $20 \mu \mathrm{L}$, in the presence of $10 \mu \mathrm{L}$ DyNAmo SYBR Green Mastermix (FinnZymes, Espoo, Finland) and $0.3 \mu \mathrm{M}$ each of a forward and a reverse primers. Primers of 20-24 nucleotides in length (usually located in the $3^{\prime}$-untranslated region) with a $T_{m}$ of $60^{\circ} \mathrm{C}$ were designed to amplify a gene-specific, 160-200 bp fragment of the target cDNA. Reactions were carried out in triplicate in an ABI7900HT RT-PCR machine (ABI, Foster City, USA)with an initial step of $3 \mathrm{~min}$ at $95^{\circ} \mathrm{C}$, followed by 40 cycles of $3 \mathrm{~s}$ at $95^{\circ} \mathrm{C}$ and $20 \mathrm{~s}$ at $60-63^{\circ} \mathrm{C}$. Each cycle was followed by a data-acquisition step. After the last cycle and a final 10 -min extension at $72^{\circ} \mathrm{C}$, a melting curve was measured from 60 to $95^{\circ} \mathrm{C}$ with measurements every $0.2^{\circ} \mathrm{C}$ and holding for $1 \mathrm{~s}$. Fluorescence was determined following each annealing and extension phase. 


\section{Availability of supporting data}

The RNA-seq data supporting the results of this article are available at the NCBI under BioProject (http://www. ncbi.nlm.nih.gov/bioproject/211928) with accession number PRJNA211928.

\section{Additional files}

\section{Additional file 1: Details of the 22,295 genes identified in six libraries of Picea balfouriana.}

Additional file 2: Enriched GO terms and KEGG pathways for the 22,295 genes identified in six libraries of Picea balfouriana.

Additional file 3: Genes selected from two-factor analysis of variance as differentially expressed between the ET and NET libraries. Additional file 4: Differentially expressed genes between the ET and NET libraries confirmed by DESeq.

Additional file 5: Enriched GO terms and KEGG pathways for the differentially expressed genes between the ET and NET libraries.

\section{Abbreviations}

AGP: Arabinogalactan proteins; ET: Embryogenic tissue; NET: Non-embryogenic tissue; FDR: False-discovery rate; Picea balfouriana: Picea likiangensis var. balfouriana (Rehd. et Wils.) Hillier ex Slavin; SERK: Somatic embryogenesis receptor-like kinase.

\section{Competing interests}

The authors declare that they have no competing interests.

\section{Authors' contributions}

JW and SZ conceived and designed the study. QL performed the experiments and wrote the manuscript. All authors read and approved the final manuscript.

\section{Acknowledgments}

Financial support for this study was provided by the Public Industrial and Special Scientific Research of Forestry, China (Project 201004009-1).

Received: 23 July 2013 Accepted: 30 June 2014

Published: 4 July 2014

\section{References}

1. Stasolla C, Kong L, Yeung EC, Thorpe TA: Maturation of somatic embryos in conifers: morphogenesis, physiology, biochemistry and molecular biology. In Vitro Cell Dev Biol-Plant 2002, 38:93-105.

2. Schuster SC: Next-generation sequencing transforms today's biology. Nat Methods 2008, 5:16-18.

3. Ansorge WJ: Next generation DNA sequencing techniques. New Biotechnol 2009, 25:195-203.

4. Huang W, Marth G: EagleView: a genome assembly viewer for next-generation sequencing technologies. Genome Res 2008, 18:1538-1543.

5. Blow N: Transcriptomics: The digital generation. Nature 2009, 458:239-242.

6. $\mathrm{t}$ Hoen PA, Ariyurek $\mathrm{Y}$, Thygesen $\mathrm{HH}$, Vreugdenhil E, Vossen RHAM, de Menezes RX, Boer JM, van Ommen GJB, den Dunnen JT: Deep sequencing-based expression analysis shows major advances in robustness, resolution and inter-lab portability over five microarray platforms. Nucleic Acids Res 2008, 36(21):e141.

7. Rosenkranz RG, Borodina T, Lehrach H, Himmelbauer H: Characterizing the mouse ES cell transcriptome with Illumina sequencing. Genomics 2008 92:187-194.

8. Hegedus Z, Zakrzewska A, Agoston VC, Ordas A, Rácz P, Mink M, Spaink HP, Meijer AH: Deep sequencing of the zebrafish transcriptome response to mycobacterium infection. Mol Immunol 2009, 46:2918-2930.

9. Morozova O, Marra MA: Applications of next-generation sequencing technologies in functional genomics. Genomics 2008, 92(5):255-264.

10. Mortazavi A, Williams BA, Schaeffer $L$, Wold B: Mapping and quantifying mammalian transcriptomes by RNA-Seq. Nat Methods 2008, 5(7):621-628.
11. Nagalakshmi U, Wang Z, Waern K, Shou C, Raha D, Gerstein M, Snyder M: The transcriptional landscape of the yeast genome defined by RNA sequencing. Science 2008, 320(5881):1344-1349.

12. Sultan $M$, Schulz $M H$, Richard $H$, Magen A, Klingenhoff A, Scherf M, Seifert M, Borodina T, Soldatov A, Parkhomchuk D, Schmidt D, O'Keeffe S, Haas S, Vingron $\mathrm{M}$, Lehrach $\mathrm{H}$, Yaspo ML: A global view of gene activity and alternative splicing by deep sequencing of the human transcriptome. Science 2008, 321(5891):956-960.

13. Xiang LX, He D, Dong WR, Zhang YW, Shao JZ: Deep sequencing-based transcriptome profiling analysis of bacteria-challenged Lateolabrax japonicus reveals insight into the immune-relevant genes in marine fish. BMC Genomics 2010, 11:472-493.

14. Nystedt B, Street NR, Wetterbom A, Zuccolo A, Lin YC, Scofield DG, Vezzi F, Delhomme N, Giacomello S, Lexeyenko A, Vicedomini R, Sahlin K, Sherwood E, Elfstrand M, Gramzow L, Holmberg K, Hällman J, Keech O, Klasson L, Koriabine M, Kucukoglu M, Käller M, Luthman J, Lysholm F, Niittylä T, Olson A, Rilakovic N, Ritland C, Rosselló JA, Sena J, et al: The Norway spruce genome sequence and conifer genome evolution. Nature 2013, 497:579-584.

15. Andersen $\mathrm{CL}$, Jensen $J \mathrm{~L}$, Orntoft TF: Normalization of real-time quantitative reverse transcription-PCR data: A model-based variance estimation approach to identify genes suited for normalization, applied to bladder and colon cancer data sets. Cancer Res 2004, 64:5245-5250.

16. Pfaffl MW, Tichopad A, Prgomet C, Neuvians TP: Determination of stable housekeeping genes, differentially regulated target genes and sample integrity: BestKeeper-Excel-based tool using pair-wise correlations. Biotechnol Lett 2004, 26:509-515.

17. Spinsanti G, Panti C, Lazzeri E, Marsili L, Marsili L, Casini S, Frati F, Fossi CM Selection of reference genes for quantitative RT-PCR studies in striped dolphin (Stenella coeruleoalba) skin biopsies. BMC Mol Biol 2006, 7:32

18. Ainley WM, Walker JC, Nagao RT, Key JL: Sequence and characterization of two auxin regulated genes from soybean. J Biol Chem 1988, 263:10658-10666.

19. Takaahashi Y, Kuroda H, Tanaka T, Machida Y, Takebe I, Nagata T: Isolation of an auxin regulated gene cDNA expressed during the transition from $\mathrm{G}_{0}$ to $\mathrm{S}$ phase in tobacco mesophyll protoplasts. Proc Natl Acad Sci U S A 1989, 86:9279-9283

20. Bögre L, Stefanov I, Abrahám M, Somogyi I, Dudits D: Differences in responses to 2,4-D-dichlorophenoxy acetic acid (2,4-D) treatment between embryogenic and non-embryogenic lines of alfalfa. In Progress in Plant cellular and Molecular Biology. Edited by Nijkamp HJJ, van der Plas LHW, Vartrajk J. Boston: Kluwer Academic Publishers; 1990:427-436.

21. Schmidt EDL, Guzzo F, Toonen MAJ, de Vries SC: A leucine-rich repeat containing receptor-like kinase marks somatic plant cells competent to form embryos. Development 1997, 124:2049-2062.

22. Somleva MN, Schmidt EDL, de Vries SC: Embryogenic cells in Dactylis glomerata L. (Poaceae) explants identified by cell tracking and by SERK expression. Plant Cell Rep 2000, 19:718-726.

23. Hecht V, Vielle-Calzada JP, Hartog MV, Schmidt EDL, Boutilier K, Grossniklaus U, de Vries SC: The Arabidopsis SOMATIC EMBRYOGENESIS RECEPTOR KINASE 1 gene is expressed in developing ovules and embryos and enhances embryogenic competence in culture. Plant Physiol 2001, 127:803-816.

24. Nolan KE, Irwanto RR, Rose RJ: Auxin up-regulates MtSERK1 expression in both Medicago truncatula root-forming and embryogenic cultures. Plant Physiol 2003, 133:218-230

25. Thomas C, Meyer D, Himber C, Steinmetz A: Spatial expression of a sunflower SERK gene during induction of somatic embryogenesis and shoot organogenesis. Plant Physiol Biochem 2004, 42:35-42

26. Santa-Catarina C, Hanai LR, Dornelas MC, Viana AM, Floh EIS: SERK gene homolog expression, polyamines and amino acids associated with somatic embryogenic competence of Ocotea catharinensis Mez. (Lauraceae). Plant Cell Tissue Organ Cult 2004, 79:53-61.

27. Shimada T, Hirabayashi T, Endo T, Fujii H, Kita M, Omura M: Isolation and characterization of the somatic embryogenesis receptor-like kinase gene homologue (CitSERK1) from Citrus unshiu Marc. Sci Hortic 2005, 103:233-238.

28. de Oliveira SM, Romano E, Yotoko KSC, Tinocoa MLP, Diasa BBA, Aragão FU: Characterization of the cacao somatic embryogenesis receptor-like kinase (SERK) gene expressed during somatic embryogenesis. Plant Sci 2005, 168:723-729. 
29. Singla B, Khurana JP, Khurana P: Characterization of three somatic embryogenesis receptor kinase genes from wheat, Triticum aestivum. Plant Cell Rep 2008, 27:833-843.

30. Shiota H, Satoh R, Watabe K, Harada H, Kamada H: C-ABI3, the carrot homologue of the Arabidopsis $A B I 3$, is expressed during both zygotic and somatic embryogenesis and functions in the regulation of embryo-specific ABA-inducible genes. Plant Cell Physiol 1998, 39:1184-1193.

31. Chapman A, Blervacq AS, Vasseur J, Hilbert JL: Arabinogalactan-proteins in Cichorium somatic embryogenesis: effect of $\beta$-glucosyl Yariv reagent and epitope localization during embryo development. Planta 2000, 211:305-314.

32. Leljak-Levanic D, Naana B, Jelaska MS: Changes in DNA methylation during somatic embryogenesis in Cucurbita pepo L. Plant Cell Rep 2004, 23:120-127.

33. Stasolla C, Bozhkov PV, Chu TM, van Zyl L, Egertsdotter U, Suarez MF, Craig D, Wolfinger RD, Von Arnold S, Sederoff RR: Variation in transcript abundance during somatic embryogenesis in gymnosperms. Tree Physiol 2004, 24:1073-1085.

34. Toonen MA, Schmidt EDL, Heo TH, Verhoeven HA, van Kammen A, de Vries SC: Expression of the JIM8 cell wall epitope in carrot somatic embryogenesis. Planta 1996, 200:167-173.

35. van der Graa VE, Dulk-Ras AD, Hooykaas PJJ, Keller B: Activation tagging of the LEAFY PETIOLE gene affects leaf petiole development in Arabidopsis thaliana. Development 2000, 127:4971-4980

36. van Hengel AJ, Guzzo F, van Kammen A, de Vries SC: Expression pattern of the carrot EP3 endochitinase genes in suspension cultures and in developing Seeds. Plant Physiol 1998, 117:43-53.

37. von Recklinghausen IR, Iwanowska A, Kieft H, Mordhorst AP, Schel JHN, van Lammeren AAM: Structure and development of somatic embryos formed in Arabidopsis thaliana pt mutant callus cultures derived from seedlings. Protoplasma 2000, 211:217-224.

38. Kreuger M, van Hoist GJ: Arabinogalactan proteins are essential in somatic embryogenesis of Daucus carota L. Planta 1993, 189:243-248.

39. Egertsdotter $U$, von Arnold S: Importance of arabinogalactan proteins for the development of somatic embryos of Norway spruce (Picea abies). Physiol Plant 1995, 93:334-345.

40. Kreuger M, Postma E, Brouwer $Y$, van Holst GJ: Somatic embryogenesis of Cyclamen persicum in liquid medium. Physiol Plant 1995, 94:605-612.

41. Saare-Surminski K, Preilb W, Knox JP, Lieberei R: Arabinogalactan proteins in embryogenic and non-embryogenic callus cultures of Euphorbia pulcherrima. Physiol Plant 2000, 108:180-187.

42. Filonova LH, von Arnold S, Daniel G, Bozhkov PV: Programmed cell death eliminates all but one embryo in a polyembryonic plant seed. Cell Death Differ 2002, 9:1057-1062.

43. Haecker A, Gross-Hardt R, Geiges B, Sarkar A, Breuninger H, Herrmann M, Laux T: Expression dynamics of WOX genes mark cell fate decisions during early embryonic patterning in Arabidopsis thaliana. Development 2004, 131:657-668.

44. Nardmann J, Zimmermann R, Durantini D, Kranz E, Werr W: WOX gene phylogeny in Poaceae: a comparative approach addressing leaf and embryo development. Mol Biol Evol 2007, 24:2474-2484.

45. Palovaara J, Hakman I: Conifer WOX-related homeodomain transcription factors, developmental consideration and expression dynamic of WOX2 during Picea abies somatic embryogenesis. Plant Mol Biol 2008, 66:533-549.

46. Klimaszewska K, Overton C, Stewart D, Rutledge RG: Initiation of somatic embryos and regeneration of plants from primordial shoots of 10-year-old somatic white spruce and expression profiles of 11 genes followed during the tissue culture process. Planta 2011, 233:635-647.

47. Hedman H, Zhu TQ, von Arnold S, Sohlberg JJ: Analysis of the WUSCHEL-RELATED HOMEOBOX gene family in the conifer Picea abies reveals extensive conservation as well as dynamic patterns. BMC Plant Biol 2013, 13:89.

48. Litvay JD, Verma DC, Johnson MA: Influence of a loblolly pine (Pinus taeda L.) culture medium and its components on growth and somatic embryogenesis of the wild carrot (Daucus carota L.). Plant Cell Rep 1985, 4:325-328.

49. Li R, Yu C, Li Y, Lam TW, Yiu SM, Kristiansen K, Wang J: SOAP2: an improved ultrafast tool for short read alignment. Bioinformatics 2009, 25(15):1966-1967.
50. Law CW, Chen Y, Shi W, Smyth GK: Voom! Precision weights unlock linear model analysis tools for RNA-seq read counts. Genome Biol 2014, 15:R29.

51. Anders S, Huber W: Differential expression analysis for sequence count data. Genome Biol 2010, 11(10):R106.

52. Benjamini $Y$, Hochberg $Y$ : Controlling the false discovery rate: a practical and powerful approach to multiple testing. J R Stat Soc Series B-Methodological 1995, 57(1):289-300.

53. Friedmann M, Ralph SG, Aeschliman D, Zhuang J, Ritland K, Ellis BE, Bohlmann J, Douglas CJ: Microarray gene expression profiling of developmental transitions in Sitka spruce (Picea sitchensis) apical shoots. J Exp Bot 2007, 58:593-614.

54. Phillips MA, D'Auria JC, Luck K, Gershenzon J: Evaluation of Candidate Reference Genes for Real-Time Quantitative PCR of Plant Samples Using Purified cDNA as Template. Plant Mol Biol Rep 2009, 27:407-416.

55. Ralph S, Yueh H, Friedmann M, Zeznik JA, Nelson CC, Butterfield YSN, Kirkpatrick R, Liu J, Jones SJM, Marra MA, Douglas CJ, Ritland K, Bohlmann J: Conifer defense against insects: microarray gene expression profiling of Sitka spruce (Picea sitchensis) induced by mechanical wounding or feeding by spruce budworms (Choristoneura occidentalis) or white pine weevils (Pissodes strobi) reveals large scale changes of the host transcriptome. Plant Cell Environ 2006, 29:1545-1570.

doi:10.1186/1471-2164-15-553

Cite this article as: Li et al:: Transcriptome analysis of callus from Picea balfouriana. BMC Genomics 2014 15:553.

\section{Submit your next manuscript to BioMed Central and take full advantage of:}

- Convenient online submission

- Thorough peer review

- No space constraints or color figure charges

- Immediate publication on acceptance

- Inclusion in PubMed, CAS, Scopus and Google Scholar

- Research which is freely available for redistribution 